\title{
Glottal Motion and its Impact on the Respiratory Flow
}

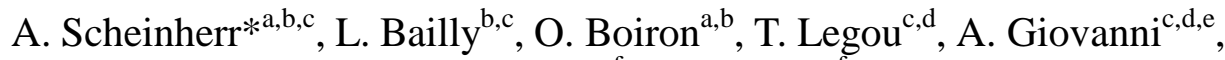 \\ G. Caillibotte ${ }^{\mathrm{f}}, \mathrm{M}$. Pichelin ${ }^{\mathrm{f}}$ \\ a. Centrale Marseille, IRPHE, 13451, Marseille, France \\ b. CNRS, IRPHE, 13384, Marseille, France \\ c. Aix-Marseille Univ, IRPHE, 13384, Marseille, France \\ d. CNRS, LPL UMR6057, Aix-en-Provence, France \\ e. Service ORL Hôpital de La Timone, CHU Marseille \\ f. Air Liquide Santé International, Groupe Gaz Médicaux, CRCD, Jouy-en-Josas, France
}

Keywords: Glottis; Breathing; Laryngofiberscopy; Image processing; CFD simulations

\section{Introduction}

Advantages of inhaled therapies as a priori targeted supply of drugs make them particularly convenient for the treatment of lung diseases. Nevertheless, several physical and anatomical factors can largely influence treatment efficiency. In particular, upper airways (UA) anatomic arrangement acts as an unwanted filter, which limits the amount of drug delivered to the lung [5]. More specifically, the glottis, defined by vocal folds aperture within the larynx, causes airways to narrow in a minimal transition cross-section. This anatomical singularity yields to a complex jet-like tracheal flow [4, 9, 11], which can be determinant on particles deposition by inertial impaction. However, current studies are limited by two main issues:

- Glottal dynamics during human breathing have been barely investigated so far, and despite a few reference in vivo studies $[1,3]$, the relationship between glottal area and inhaled airflow is still poorly understood.

- The aerodynamic influence of the glottal geometrical changes during breathing is often discarded in numerical works. Instead, a static glottis is often considered together with steady flow conditions [4, 9]. Therefore, the aim of the present study is (i) to characterize the glottal dynamics during human breathing in vivo using laryngofiberscopy and synchronized airflow recordings; (ii) to quantify the effects of a mobile glottis and unsteady flow conditions on laryngeal jet-flow dynamics using CFD modeling.

\section{Methods}

In vivo study. In vivo experiments were conducted in the ENT department of La Timone Hospital. One healthy female volunteer (LB, age 29) and one healthy male volunteer (OB, age 48) were recorded while performing two 30s breathing tasks: normal breathing (eupnea) and forced breathing (tachypnea). Laryngofiberscopic investigations were made using a flexible nasofiberscope (Storz 20222020 tricam camera) with a continuous cold light source and a color CCD camera. Laryngeal images were captured with a camera frame rate of $25 \mathrm{frames} / \mathrm{s}$ and an image resolution of $768 \times 288$ pixels. The oral airflow signal was simultaneously registered by means of a pneumotachograph placed at the mouth, EVA2 [7].

In silico study. As a first approximation of the glottal geometry was built a $2 \mathrm{D}$ rectangular moving constriction with a triangular dynamic mesh of 24000 el. The mesh density ensures grid-independent results. CFD simulations were conducted using Fluent 6.3.26 under laminar air-flow conditions, assuming an incompressible Newtonian gas of viscosity $v$ equal to $1.789 \cdot 10^{-5} \mathrm{~kg} / \mathrm{ms}$. A pressure outlet boundary condition was set to $0 \mathrm{~Pa}$. Unsteady boundary conditions comprising the glottal width $\mathrm{d}_{\mathrm{g}}(\mathrm{t})$ and the velocity inlet were parametrically varied in agreement with the in vivo study. A no-slip shear boundary condition was applied at solid walls. Initially, zero velocities and pressures were assumed at all points. Equations were solved using a first-order time and spatial discretization schemes, and a time step set to $0.12 \mathrm{~s}$.

\section{Results and discussion}

Airflow rate. In vivo measurements yielded to about 30 respiratory cycles during eupnea and 40 during tachypnea. Every respiratory cycle was detected using a zero-tracking method developed in Matlab R2011b. Each airflow signal $\mathrm{Q}$ was normalized with respect to the maximum value achieved within the cycle, $\mathrm{Q}_{\max }$. Time $\mathrm{t}$ was normalized by corresponding respiratory period, $\mathrm{T}=2 \pi / \omega$. The maximal period registered within each $30 \mathrm{~s}$ sequence is noted $\mathrm{T}_{\max }$. Signals were finally averaged into one mean flow-rate, herein noted $\left.<\mathrm{Q} / \mathrm{Q}_{\max }\right\rangle$. Figure 1a illustrates the typical flow-rate $<\mathrm{Q} / \mathrm{Q}_{\max }>$ as a function of $\omega \mathrm{t}$, produced by subject $\mathrm{OB}$ during eupnea (maximal $\mathrm{Q}_{\max }=1.9 \mathrm{dm}^{3} / \mathrm{s}, \mathrm{T}_{\max }=$ $0.62 \mathrm{~s}$ ) and tachypnea (maximal $\mathrm{Q}_{\max }=4.3 \mathrm{dm}^{3} / \mathrm{s}, \mathrm{T}_{\max }$ $=4.8 \mathrm{~s})$. In comparison, a sinusoidal evolution is plotted. Conventionally, positive (resp. negative) flowrate values correspond to expiration (resp. inspiration) phase. During eupnea, the inspiration and expiration curves are similar (up to sign) and their durations are approximately equal, as commonly found in the literature [6]. During tachypnea, the mean flow-rate curve deviates from the harmonic signal although inspiration and expiration durations remain roughly equal. A phase difference of about $22^{\circ}$ in flow-rate maximal occurrences has been measured between sinusoid and eupnea curve, which corresponds to $6 \%$ of the breathing period. The phase difference increases during tachypnea up to $58^{\circ}$, namely $16 \%$ of the cycle duration. LB breathing shows similar characteristics. 
Glottal motion. Glottal motion was extracted from the laryngoscopic images using Matlab Image Processing Toolbox and different phases: (i) Focus on a region of interest using a cross-correlation technique, (ii) Smoothing using a specific filter function [10], (iii) Detection of the glottal contours using a segmentation method [2] (iv) Measurement of the glottal anteroposterior diameter $\mathrm{AP}_{\mathrm{g}}$, glottal area $\mathrm{A}_{\mathrm{g}}$ and glottal width $d_{g}$ (see Fig. 1b), (v) Normalization by comparing $\mathrm{AP}_{\mathrm{g}}$ diameter with the initial value $\mathrm{AP}_{\mathrm{g}}{ }^{0}$, assumed as a geometrical invariant [8], (vi) Conversion from pixels to millimeters, assuming $\mathrm{AP}_{\mathrm{g}}=22 \mathrm{~mm}$ [5].

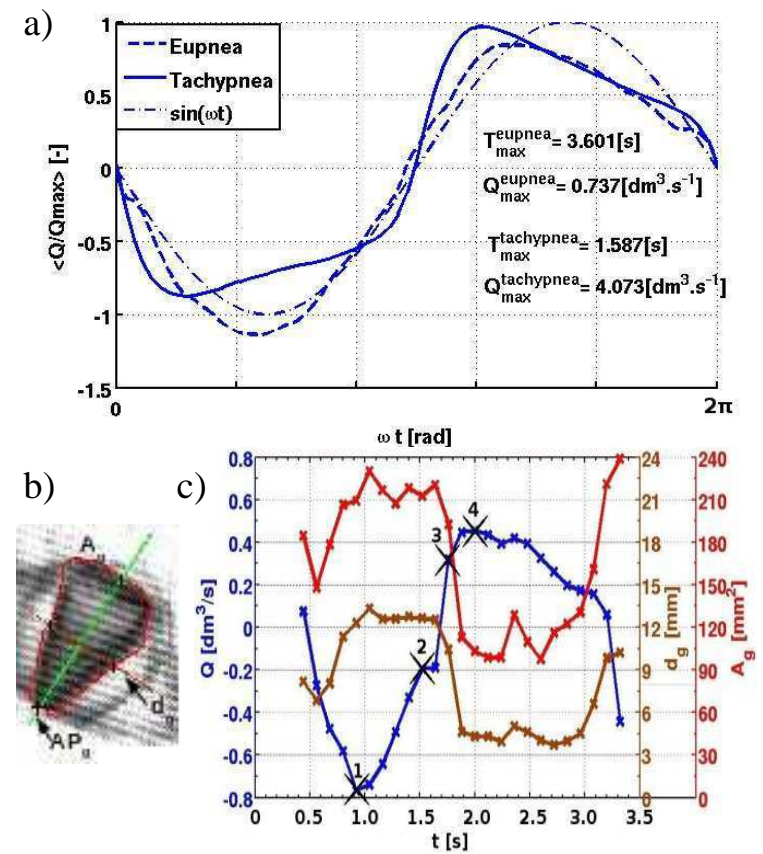

Figure 1: (a) Mean flowrate $<\mathrm{Q} / \mathrm{Qmax}>$ measured during cycles of eupnea and tachypnea (subject

OB) and comparison with sinusoid. (b) Illustration of glottal image post-processing.(c) Detected glottal area $\mathrm{Ag}$ and glottic width dg and flowrate Q.

Fig. 1c illustrates the evolution of the glottal dynamics measured during a typical eupnea cycle. Glottal area $\mathrm{A}_{\mathrm{g}}$ and glottal width $\mathrm{d}_{\mathrm{g}}$ are displayed as function of time, together with synchronized airflow-rate Q. It is shown that the glottis progressively widens during inspiration and narrows during expiration. Area $\mathrm{A}_{g}$ varies in the range $90-240 \mathrm{~mm}^{2}$ (mean value $165 \mathrm{~mm}^{2}$ ) during the cycle, while $d_{\mathrm{g}}$ varies in the range $4-13 \mathrm{~mm}$ (mean value $7.8 \mathrm{~mm}$ ), thus achieving a peak value during inspiration nearly three times greater than that measured during expiration. This ratio is in line with previous studies $[1,3]$. Above measurements allowed to assess a Reynolds number $\left(\operatorname{Re}=\left\{\mathrm{d}_{\mathrm{g}} \mathrm{Q}\right\} /\left\{v \mathrm{~A}_{\mathrm{g}}\right\}\right)$ ranging from 500 to 2500 .

CFD simulations. Unsteady flow simulations were conducted considering $\mathrm{Q}$ and $\mathrm{dg}$ measured during the typical eupnea cycle (Fig. 1c). Fig. 2 shows the development of the glottal jet at 4 shot-instants at first during inspiration and consequently during expiration phase of breathing (crosses in Fig. 1c). Inertial effects associated to flow-rate variations yield to the jet instability and fluctuations of the reattachment area during the breathing cycle but the flow despite $\mathrm{Re} \cong 2500$ stays laminar as already noticed in [12].

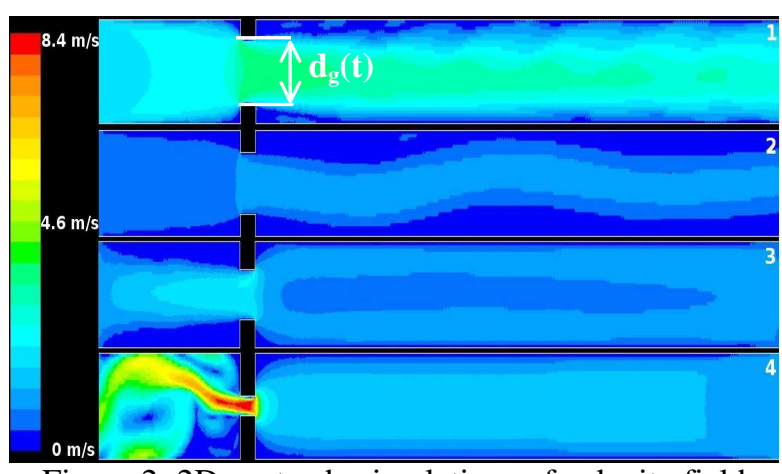

Figure 2: 2D unsteady simulations of velocity field through the moving glottis during a breathing cycle.

\section{Conclusions}

The in vivo study showed that the glottis can be extremely variable during breathing and hence influence airflow characteristics. A glottal area widening was quantified during inspiration, with a typical ratio of 3:1 as compared to expiration. Airflow rate variations differ from harmonic signal during eupnea as well as tachypnea. The correlation between flow-rate and glottal area will be discussed and compared to previous clinical investigations. Preliminary 2D CFD simulations of the glottal jet were performed, based on the measured flow-rate and glottal changes during eupnea. Impact of unsteady flow conditions on the jet development is demonstrated.

\section{References}

[1] Baier, H., Wanner, A., Zarzecki, S., Sackner, M.A., 1977. Relationships among glottis opening, respiratory flow and upper airway resistance in humans. J Appl Physiol, 43, 603-611.

[2] Bernard O., Friboulet D., Thévenaz P., Unser M., 2009. Variational B-Spline level-set: A linear filtering approach for fast deformable model evolution. IEEE Trans. Image Process, 18, 1179-1191.

[3] Brancatisano T., Collett P., Engel L., 1983. Respiratory movements of the vocal cords. J Appl Physiol, 54 (5), 1269-1276.

[4] Brouns M., Verbanck S., Lacor C., 2007. Influence of glottal aperture on the tracheal flow. J Biomech, 40, 165-172.

[5] Conway et al., 2012. Controlled, Parametric, Individualized, 2-D and 3-D Imaging Measurements of Aerosol Deposition in the Respiratory Tract of Healthy Human Subjects for Model Validation. J Aerosol Sci submitted.

[6] Fenn W. O., Rahn H., 1965. Handbook of Physiology, Section 3: Respiration, Ame Physio Soc, Washington, DC.

[7] Ghio A., Teston B., 2004. Evaluation of the acoustic and aerodynamic constraints of a pneumotachograph for speech and voice studies. ICVPB, Marseille, France, 55-58.

[8] Higenbottam T., 1980. Narrowing of glottis opening in humans associated with experimentally induced bronchoconstriction. J Appl Physiol, 49, 403-407.

[9] Katz, I., Martonen, T., Flaa, W., 1997. Threedimensional computational study of inspiratory aerosol flow through the larynx: the effect of glottal aperture modulation. $J$ Aerosol Sc 28, 1073-1083.

[10] Kroon D.J., 2009. Hessian based Frangi Vesselness filter Matlab code. University of Twente, Hollande.

[11]Renotte C., Bouffioux V., Wilquem F., 2000. Numerical analysis of oscillatory flow in the time-varying laryngeal channel. J. Biomech, 33, 1637-1644.

[12] Boiron O., Deplano V., Pelissier R., 2007. Experimental and numerical studies on the starting effect on the secondary flow in a bend. JFM, 574, 109-129. 